\title{
Le goyavier rouge : Psidium cattleyanum var. coriaceum (Mart. ex 0 . Berg) Kiaersk. une espèce envahissante, à usage multiple en Grande-Comore
}

\author{
Mohamed Mahamoud Charahabil \\ (Maître Assistant, PhD, Écologue - Botaniste) \\ Département d'Agroforesterie, \\ Université Assane Seck de Ziguinchor/Sénégal \\ Leonard Elie Akpo \\ (Professeur Titulaire des Universités, Écologue) \\ Laboratoire d'Écologie et d'écohydrologie, \\ Département de Biologie végétale, Université Cheikh Anta Diop de Dakar
}

Doi: 10.19044/esj.2018.v14n9p436 URL:http://dx.doi.org/10.19044/esj.2018.v14n9p436

\begin{abstract}
The geographical position of the Comoros archipelago conditions an environment conducive to continental biological invasions. In the current context of climate change, with the repeated arrival of strong winds from nearby coasts, the phenomenon is likely to worsen. This study presents the characteristics of the biological invasion of Psidium. cattleyanum var. Coriaceum in Grande-Comore using quantitative structural data of its population and modeling of its ecological niche. The uses of the species and its socio-economic importance were defined by means of a population survey. The overall results revealed the species' invasion status through density, relative frequency, regeneration potential and horizontal distribution model. The modeling of its ecological niche has shown that all the wooded areas of the island are potential sites of the species. Socioeconomic surveys have shown that Psidium. Cattleyanum is a multipurpose species well appreciated by the people. The fight against this invasion very common to all the islands of the southwest of the Indian Ocean faces, in this island of the archipelago of Comoros, a conflict of interest between the conservation of biodiversity and satisfaction the needs of these populations. This work provided reference frames that would allow a better knowledge of the phenomenon for better management.
\end{abstract}

Keywords: Ecological niches, Comoros, invasion, conservation, biodiversity, Psidium. cattleyanum var. coriaceum 


\section{Résumé}

La position géographique des îles Comores conditionne un environnement propice aux invasions biologiques continentales. Dans le contexte actuel de changement climatique, avec les arrivées répétées des vents violents venant des côtes voisines, le phénomène risque de s'aggraver. Cette étude présente les caractéristiques de l'invasion biologique du Psidium. cattleyanum var. Coriaceum en Grande-Comore à l'aide de données structurales quantitatives de sa population et d'une modélisation de sa niche écologique. Les usages de l'espèce et son importance socioéconomique ont été définis à l'aide d'une enquête réalisée auprès des populations. L'ensemble des résultats a mis en évidence le statut d'envahissement de l'espèce à travers sa densité, sa fréquence relative, son potentiel de régénération et son modèle de distribution horizontale. La modélisation de sa niche écologique a montré que tous les espaces boisés de l'île sont des sites potentiels de l'espèce. Les enquêtes socioéconomiques ont montré que Psidium Cattleyanum est une espèce à usages multiples bien appréciée par les populations. La lutte contre cette invasion très commune à l'ensemble des iles du sud-ouest de l'océan indien est confrontée, dans cette île de l'archipel des Comores, à un conflit d'intérêts entre la conservation de la biodiversité et la satisfaction des besoins de ces populations. Ce travail fourni des référentiels qui permettraient une meilleure connaissance du phénomène pour une meilleure gestion.

Mots-clefs: Niches écologiques, Comores, invasion, conservation, biodiversité, Psidium. cattleyanum var. coriaceum

\section{Introduction}

La Grande-Comore présente un intérêt exceptionnel en termes de diversité biologique, du fait de l'endémisme important de sa flore dans une île, jeune (100 000 à 130000 ans) et de taille réduite $\left(1025 \mathrm{~km}^{2}\right)$. Par rapport aux autres îles de l'archipel des Comores, elle est la première à être peuplée par l'homme du fait de sa proximité avec la côte africaine (Vos, 2004). Il semblerait que la première vague de population africaine originaire de la côte orientale d'Afrique a atteint les Comores à l'âge du fer, entre le $\mathrm{V}^{\mathrm{e}}$ et le $\mathrm{X}^{\mathrm{e}}$ siècle. C'est à partir du XVIe siècle que l'on note l'arrivée aux Comores d'une nouvelle vague d'Arabo-Shirazi (Rombi, 2018). C'est entre le XVIe et XVIIe siècle que des navigateurs européens, en route vers les Indes, font escale aux Comores (Liszkowski, 2000). Les premières introductions de plantes ont commencé en ces périodes. Il fallait cependant, attendre trop tardivement (mis en valeur des terres par les Français au 19e siècle) pour voir l'influence de ces espèces introduites sur la flore locale. 
Ces introductions ont causé des invasions biologiques dont le cas le plus connu et le plus problématique est celui du Psidium cattleyanum coriaceum (Mtsongoma en langue locale). Cette espèce exotique s'est en effet naturalisée dans de nombreux milieux insulaires tropicaux et subtropicaux du globe où il a un comportement de peste végétale (Lorence et Sussman, 1986; Huenneke et Vitousek, 1990 ; Fleischmann, 1997). Sa production importante de graines au taux de germination élevé, son adaptation à une large gamme écologique, sa facilité de dissémination par les oiseaux et les mammifères, et l'absence de ravageurs en font une espèce agressive et envahissante qui colonise différents milieux et empêche le développement de la flore indigène (Huenneke et Vitousek, 1990). Elle atteint des proportions impressionnantes dans l'ensemble des îles du sud-ouest de l'océan indien (Baider \& Florens, 2001 ; Carrière et al., 2008; Tassin et al., 2009 a, Tassin et al., 2009 b). Cependant, l'information sur les espèces envahissantes dans l'archipel des Comores est encore peu connue comparée à l'information disponible à la Réunion ou l'île Maurice (Tassin et al., 2006). D'après les travaux de (Vos, 2004), l'archipel des Comores compte 16 espèces ligneuses envahissantes. Ces espèces ont été introduites dans l'archipel comme bois d'œuvre, espèces fruitières, épices, pour le contrôle de l'érosion, comme ornementales, pour leurs multiples usages tels que bois de chauffe, fourrage et/ou tuteurs de vanilles, ou pour des raisons incertaines. Parmi ces 16 principales espèces envahissantes, on en distingue 8 très problématiques et qui sont ou devraient être une priorité de gestion: Acacia mangium, Acacia auriculiformis, Clidemia hirta, Lantana camara, Litsea glutinosa, Psidium cattleianum, Spathodea campanulata et Syzygium jambos. Toutes ces espèces occupent vigoureusement les zones perturbées ou les forêts secondaires, mais semblent également à même d'envahir les habitats intacts où elles ont été rencontrées sur le terrain. Au Karthala en versant ouest, entre les zones de cultures envahies et la forêt naturelle s'est développé un taillis dense monospécifique de $P$. cattleianum qui représente un foyer néfaste pour l'envahissement progressif.

La problématique de la gestion de l'invasion du $P$. cattleyanum en Grande-Comore est assez particulière. En effet, avec ses multiples usages, elle présente un très grand intérêt pour les populations. En dehors de la consommation de ses fruits, l'espèce est utilisée pour la qualité de son bois dans la construction des habitations, dans la production de charbon et dans la médecine traditionnelle (Zainaba, 2009). Elle sert aussi de tuteur pour la culture de la vanille en basse altitude.

Ses services sont accentués par la pauvreté récurrente et les perpétuelles crises énergétiques que connait l'archipel des Comores depuis plus d'une décennie. 
Dans les programmes de gestion et de conservation de la phytodiversité, les Comores projetteraient d'ériger la forêt du Karthala en aire protégée et plus tard en réserve de biosphère. Or, la dynamique d'invasion de cette espèce très particulièrement, ainsi que d'autres espèces de la strate herbacée telles que Clidemia hirta, Trisetma virusanum, et Scleria novaehollandiae (Charahabil, 2011), font de cette forêt, un biome très instable qui risque d'être colonisés par ces espèces envahissantes au détriment de la flore autochtone. Dès lors, les stratégies de lutte deviennent difficiles et sont confrontées à un problème de choix entre la protection de la diversité biologique et la satisfaction des besoins des populations (Carrière et al., 2008).

Cette étude cherche à définir les attributs écologiques qui caractérisent cette invasion à travers des données structurales quantitatives de la végétation et une modélisation de la niche écologique. Des données essentielles dans la mise en place des politiques de gestion efficace et durable des espaces boisés de l'archipel des Comores.

\section{Matériel et méthodes \\ Présentation de l'espèce \\ Botanique}

Psidium cattleyanum est un arbuste atteignant $8 \mathrm{~m}$ de hauteur. Elle appartient à la famille Myrtacées et l'ordre des Myrtales. Son écorce grise à brun rougeâtre se détache en fines plaques qui s'enroulent sur elles-mêmes. Ses feuilles sont simples et opposées, et mesurent $8 \mathrm{~cm}$ de long. Elles sont elliptiques ou oblongues, à marge entière, épaisse et brillante. Ses fleurs (Photo 1) solitaires à l'aisselle des feuilles. Elles mesurent $2,5 \mathrm{~cm}$, portent 4 à 5 pétales et sont de couleur blanche avec de nombreuses étamines blanches et jaunes (Carrière et al., 2008).

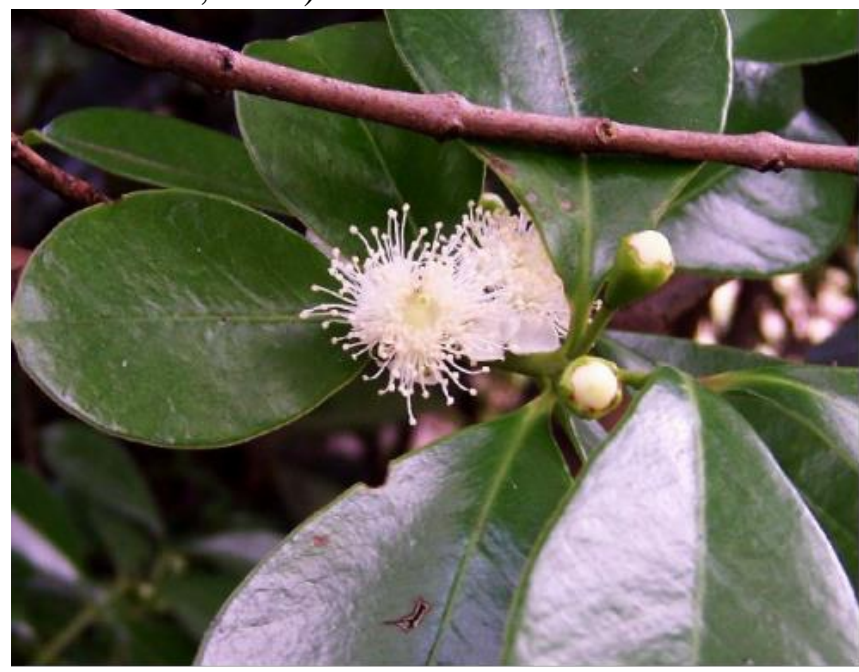

Photo 1: Fleur et boutons floraux du P. cattleyanum 
Le fruit est appelé goyave-fraise aux Antilles, goyave de Chine à l'île Maurice, goyavier à La Réunion ou Tsongoma aux Comores. Il a la forme d'une baie sphérique et la taille d'une mirabelle environ $2,5 \mathrm{~cm}$ de diamètre (Norman, 2002).

Elles sont rouges à maturité avec une chair blanchâtre et de graines nombreuses lisses et jaunâtres mesurent près de $5 \mathrm{~mm}$ de long (photo 2).

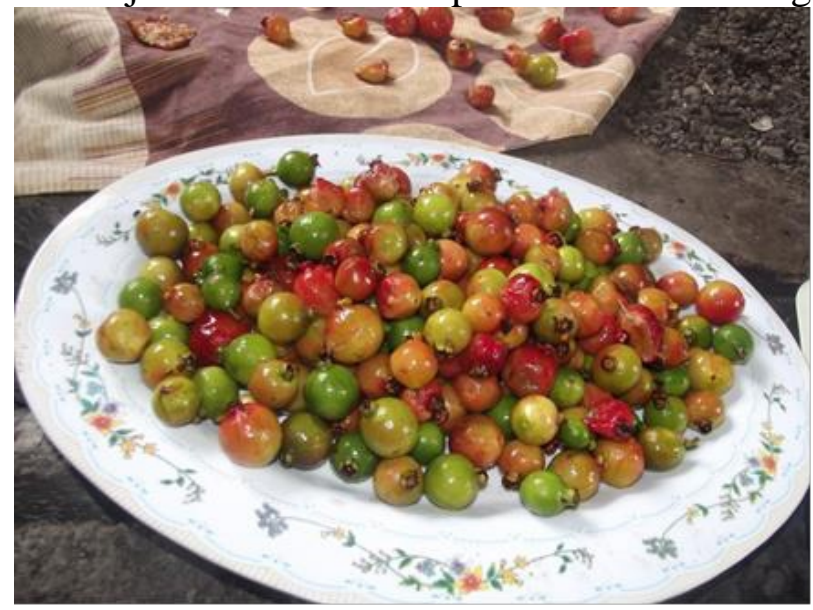

Photo 2: Fruit de P. Cattleyanum (matures en rouges et immatures en vertes).

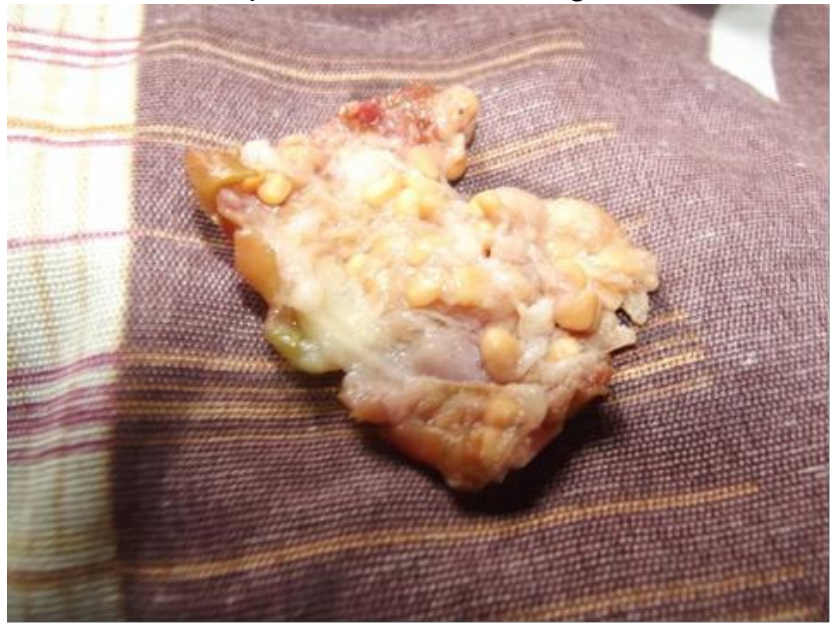

Photo 3: Fruit montrant la chaire et les graines

\section{Multiplication et mode de dissémination}

La multiplication se fait par semis important des graines (photo 4), et par bouturage. Les graines sont disséminées par la plupart des animaux, notamment plusieurs oiseaux exotiques envahissants comme le merle de Maurice. L'homme est également un vecteur important de propagation. La plante est adaptée à la plupart des milieux qu'il s'agisse de forêts naturelles ou plantées, de broussailles, de prairies, de zones dégradées ou urbaines (Carrière et al., 2008). 


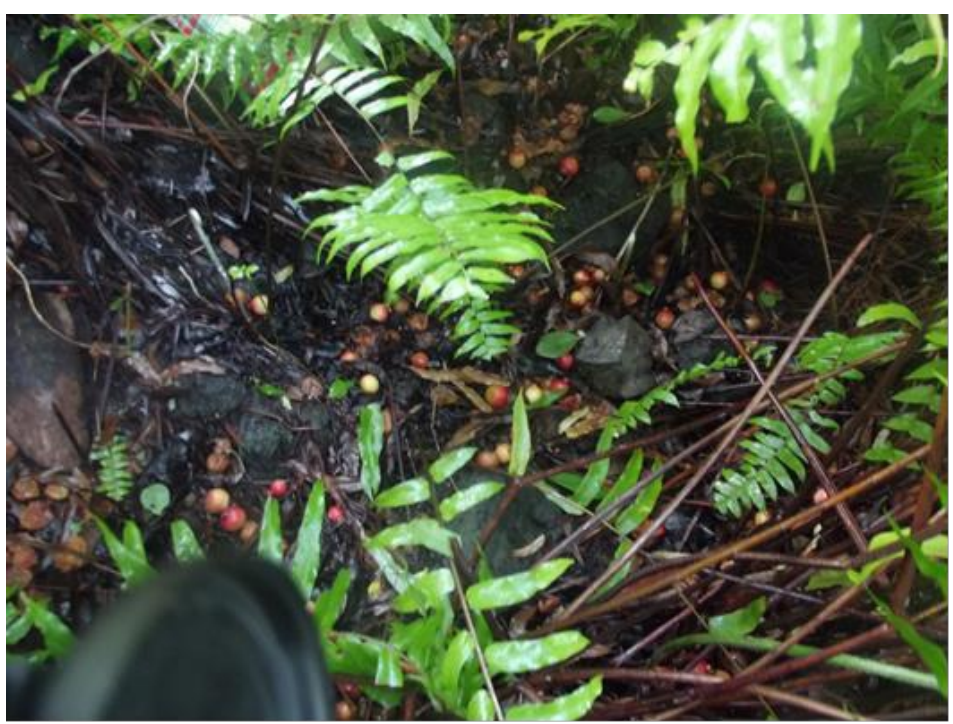

Photo 4. Quantité importante de fruits au sol témoignant du potentiel de dissémination de l'espèce.

\section{Méthode d'étude.}

\section{Inventaires floristique}

Des relevés de la végétation ont été effectués le long des sentiers pédestres dans les différents flancs environnant le Karthala et suivant le gradient altitudinal. Pour mieux embrasser les limites phytogéographiques prédéfinies, les relevés phytoécologiques ont été espacés de 100 à $200 \mathrm{~m}$ d'altitude sur chaque transect. La surface de chaque relevé est de $100 \mathrm{~m}^{2}$, équivalent à l'aire minimale de la zone d'étude définie par (Campbell et al., 1989). Au total 14 transects totalisant 78 relevés ont été délimités (Charahabil et al., 2013). Les inventaires floristiques ont été réalisés selon la méthode phytoecologique (Gounot, 1969). Au niveau de chaque placeau, la liste floristique est établie en présence-absence.

Structure horizontale

Un comptage exhaustif des ligneux dont le Dbh (Diameter Breast Height) est supérieure $30 \mathrm{~cm}$ a été ensuite réalisé dans chaque parcelle. Les individus dont le Dbh est inférieur à $30 \mathrm{~cm}$ sont considérés comme les jeunes plants issus de la régénération naturelle. Pour chaque individu adulte, le diamètre à $30 \mathrm{~cm}$ du sol a été mesuré. 
Le traitement de ces données a permis de calculer :

- La densité relative du P. Cattleyanum (Nombre d'individus de l'espèce sur le nombre total des individus* 100),

- $\quad$ La fréquence relative (Nombre de relevés où l'espèce est présente sur nombre total de relevés* 100)

- Le potentiel de régénération (Nombre de jeunes plants de l'espèce sur le nombre total de jeunes plants).

La moyenne de graines par fruit a été évaluée sur un échantillon de 50 fruits et le nombre moyen de fruits par kilogramme a été compté sur dix pesées. En fin la distribution de l'espèce par classe de grosseur a été établie.

\section{Modélisation de la niche écologique}

- Principe de la modélisation

Pour modéliser la niche écologique de l'espèce, un jeu de données de calibration associant une liste d'occurrence de l'espèce à des valeurs de paramètres environnementaux sur une centaine de points au niveau de la forêt du Karthala a été utilisé. Il s'agit de données de présence/absence sur les parcelles d'inventaires. Des extraits récents de la base Sonnerat de l'herbier de Paris ont été utilisés pour compléter le jeu de données. Les données environnementales utilisées couvrent l'ensemble de la Grande-Comore et sont facilement accessibles. Elles ont été téléchargées d'une part, sur le site de surveillance géologique des USA :

http://edc.usgs.gov/products/elevation/gtopo30/hydro/indexhtml. (Pente, indice topographique, aspect) et d'autre part sur la base de données Bioclim : http://cres.anu.edu.au/outputs/anuclim/doc/bioclim.html pour les variables climatiques (température et précipitations). Ces données sont ensuite transformées en mode raster pour être introduites dans un système d'information géographique (SIG) en tant que couche d'information (Jiguet et al., 2010). La modélisation était réalisée sous le logiciel Maxent.

\section{- Description des données environnementales}

La pente (Slopcom) exprime la variation de l'altitude mesurée en degrés entiers et variant entre 0 et $90^{\circ}$.

L'indice topographique (Topocom) communément appelé indice d'humidité qui permet d'évaluer le taux de rétention de l'eau dans un endroit donné en fonction de la pente.

L'aspect (Aspectcom) indique la direction de la plus grande pente s'exprime en degrés entiers positifs et varie en 0 et $360^{\circ}$.

La température varie très peu dans l'île et dans la forêt particulièrement. Pour maximiser son action, nous avons ainsi considéré les températures moyennes annuelles (bioccom1), les températures saisonnières (bioccom4) et les températures des mois les plus chauds (bioccom5). Les températures des mois les plus froids ont été insignifiantes et ont donc été retirées du modèle. 
Les précipitations sont généralement abondantes dans cette forêt tropicale humide, sont retenues ici, les précipitations moyennes annuelles (bioccom12), celles des mois les plus sèches (bioccom14), celles des mois les plus humides (bioccom13) et le coefficient de variation de la pluviométrie annuelle (bioccom15).

\section{- Validation du modèle produit}

La validité du modèle a été évaluée à l'aide de la courbe ROC (receiver operating characteristic), (Perneger \& Perrier 2004). Cette courbe s'interprète par la forme et par la valeur AUC qui estime le taux de discrimination entre le modèle et la réalité (Rioult, 2011). L'aire sous la courbe (ou Area Under the Curve - AUC) est un indice synthétique calculé pour les courbes ROC. L'AUC correspond à la probabilité pour qu'un événement positif soit classé comme positif par le test sur l'étendue des valeurs seuil possibles. Pour un modèle idéal, on a $\mathrm{AUC}=1$ (ci-dessus en bleu), pour un modèle aléatoire, on a $\mathrm{AUC}=0.5$ (ci-dessus en rouge). On considère habituellement que le modèle est bon dès lors que la valeur de l'AUC est supérieure à 0,7 . Un modèle bien discriminant doit avoir une AUC entre 0,87 et 0,9 .

\section{Usages de l'espèce}

Les enquêtes ethnobotaniques ont consisté en des entretiens menés au niveau des populations résidant dans 13 villages environnants du Karthala. Les données ont été recueillies à l'aide d'un questionnaire de type directif et semi-directif. Pour chaque village des exploitants potentiels de la forêt et des utilisateurs des plantes de la zone ont été questionnés. Pour compléter les données, nous avons réalisé quelques enquêtes informelles sous forme des discussions avec des chefs de villages et des Mwalimou (connaisseur et spécialistes du monde invisible des «Djinns ").

Les savoirs faire et les perceptions locaux par rapport à l'évolution des espèces utilisées seront évalués globalement et les variabilités seront recherchées en fonction de l'âge, de l'activité principale et de la localité des personnes enquêtées.

Nous avons questionné en majorité des hommes (98\%). Le choix est dicté par la disponibilité des hommes à répondre à nos questions et par leurs connaissances que l'on suppose plus solides que chez les femmes.

Les villages parcourus pour réaliser les enquêtes sont situés aux alentours du Karthala. Au Sud, on a Dzahadjou, Djoumoichongo, Mdjoiezi à l'est Kourani, kandzilé, Nioumamilima, Idjikoundzi, Ntsinimopanga, Bandadawani et à l'Ouest Bahani, Irohé, Mkazi et Nvouni.

Au total 69 personnes ont été questionnées, sur les 13 villages environnants du Karthala. Soit, en moyenne 5 personnes par village. Elles ont répondu à 147 questions réparties en six (6) thèmes que sont, 
l'alimentation humaine, l'alimentation animale, la pharmacopée, le bois de chauffage, le bois de service et les rites.

Les données d'enquête ont été d'abord dépouillées manuellement puis saisies et traitées dans le logiciel Sphinx. Les résultats ont été transformés sur le tableur Excel et ont permis de calculer les paramètres suivants :

- La Fréquence de citation qui exprime le degré de citation d'une espèce parmi les informateurs.

$$
F C=\frac{\text { Nombre de citation d'une espèce }}{\text { Nombre total de répondants }} \times 100
$$

- L'indice d'utilisation de Phillips et Gentry (1993) appelé aussi indice de préférence qui permet d'valuer l'importance de l'utilisation des plantes. Cet indice est calculé d'après la formule :

$$
\text { UVs }=\text { UVis/ns }
$$

Avec UVs : indice d'utilisation, UVis : nombre total de réponses obtenues, ns : nombre total de personnes enquêtées. Elle varie ente 0 et 1.

\section{Résultats}

\section{Données d'inventaire}

La flore ligneuse inventoriée est riche de 95 espèces réparties entre 82 genres et 45 familles. L'analyse de la fréquence relative des espèces a montré que $P$. Cattleyanum est l'espèce la plus fréquente $(57,7 \%)$ survis de Ocotea comoriensis (55,1\%), Nuxia pseudodentata (47,4\%), et Tambourissa comorensis $(46,1 \%)$. La densité relative évaluée au nombre d'invendus de l'espèce sur le nombre d'individus total a montré que $P$. Cattleyanum est largement l'espèce la plus abondante avec une densité relative de 19,55\%. Elle est suivie de Ocotea comoriensis (7,5\%,), Tambourissa comorensis (5,8\%), Weinmannia comorensis (5,5\%) et Aphloia theaeformis (3,6\%).

\section{Potentiel de génération du P. Cattleyanum}

Le nombre total de jeunes plants est de 3852 sur l'ensemble de 78 relevés effectués. $P$. Cattleyanum compte à elle seule 1399 jeunes plants soit $36,31 \%$ de régénération. $T$. comorensis présente un potentiel de régénération de $3,37 \%$, O. comoriensis $1,37 \%$, A. Theaeformis, $1,29 \%$ et $W$. comorensis, $0,49 \%$. Le potentiel de régénération d'une espèce est aussi caractérisé par le nombre de fruits produits, la quantité de graine et le pouvoir de dissémination. Le nombre moyen de fruits est estimé à 209,6 sur une 
repetition de dix pesées. Sur un échantillon de cinquante fruits étudiés, la moyenne de graines est évaluée à 30,31 graines par fruit.

\section{Strucuture horizontale du P. Cattleyanum}

La répartition des individus de l'espèce par classe de grosseur (figure 1) est un outil qui permet de décrire la distribution spatiotemporelle de l'espèce et la dynamique de sa population. Pour le cas présent, deux classes extrêmes sont les mieux représentées. Il s'agit de la classe de circonférences $(20-30 \mathrm{~cm})$ et celle des circonférences supérieures à $100 \mathrm{~cm}$. Cette configuration donne à la courbe une forme en cuvette et renvoie à un peuplement déséquilibré. Cette configuration renseigne que les jeunes plants arrivent difficilement à maturité. Ils sont coupés ou élagués avant l'âge adulte. Seuls quelques vieux arbres sont conservés dans les champs et parcours.

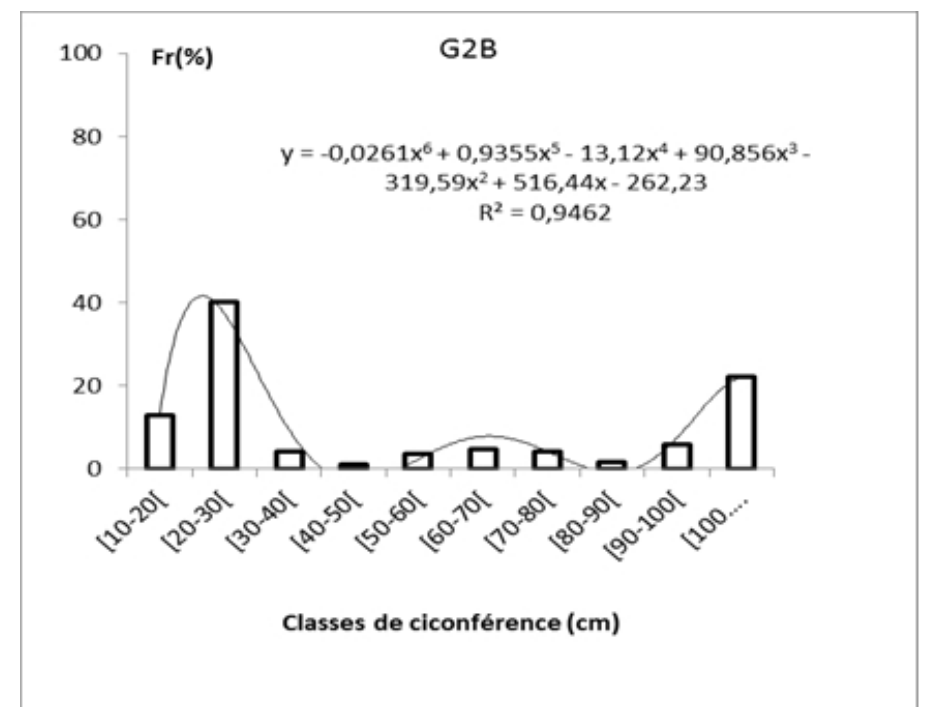

Figure 1. Histogramme de la structure par classe de grosseur du P. Cattleyanum dans la forêt de basse altitude.

\section{Modélisation de la niche écologique de l'espèce}

«Une invasion et son contrôle sont essentiellement une question de distribution dans l'espace de la plante invasive, il est fondamental que cette distribution soit étudiée et connue précisément ». La distribution spatiale et potentielle du $P$. cattleyanum var. coriaceum sont établies (Figure 2 ) à l'aide de la modélisation des niches écologiques. La carte produite par ce modèle présente des points blancs qui indiquent les sites d'observations utilisés dans le modèle et la coloration représente la répartition de la niche écologique de l'espèce. Plus la couleur est chaude (rouge) plus la prédiction est élevée. Dans 
le cas contraire (couleur bleue), les conditions environnementales ne sont pas favorables à la présence de l'espèce.

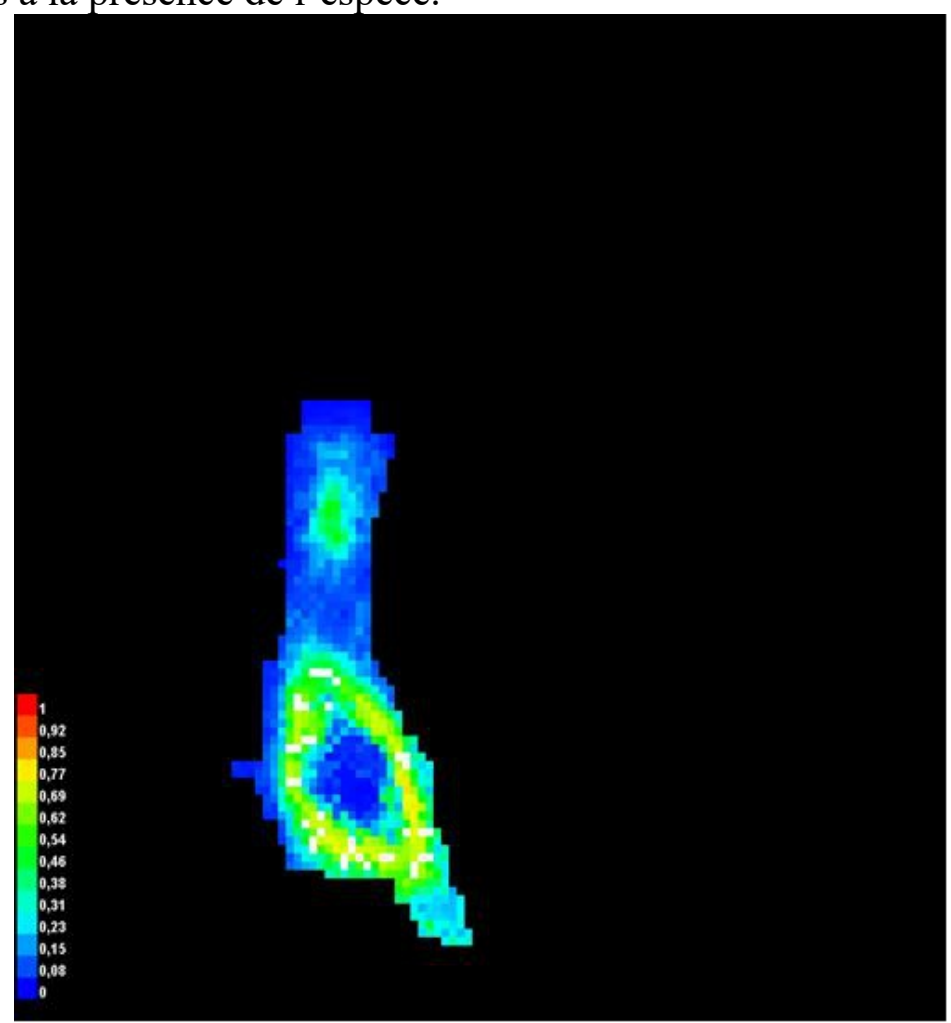

Figure 2. Carte de la niche écologique du P. cattleyanum var. Coriaceum

La carte de la niche écologique montre que toute la forêt du Karthala sont des sites potentiels de présence du $P$. cattleyanum var. coriaceum à l'exception du sommet du volcan (au-delà de $1800 \mathrm{~m}$ d'altitude) et de la bande de savanes correspondant au plateau de Dibwani qui relie le Karthala et le massif de la grille situé au nord de l'île. Dans cette forêt de la grille hautement dégradée, les prédictions de présence et donc d'envahissement avoisinent les $60 \%$. Les proportions actuelles de l'espèce dans le site restent à vérifier sur le terrain. Les zones de prédiction varient aussi suivant les versants de la forêt. À l'ouest, les probabilités de présence sont importantes à des altitudes plus élevées qu'au sud et à l'est. La forme de la courbe de ROC et la valeur AUC correspondante indiquent que le modèle est fiable (Figure 3). 


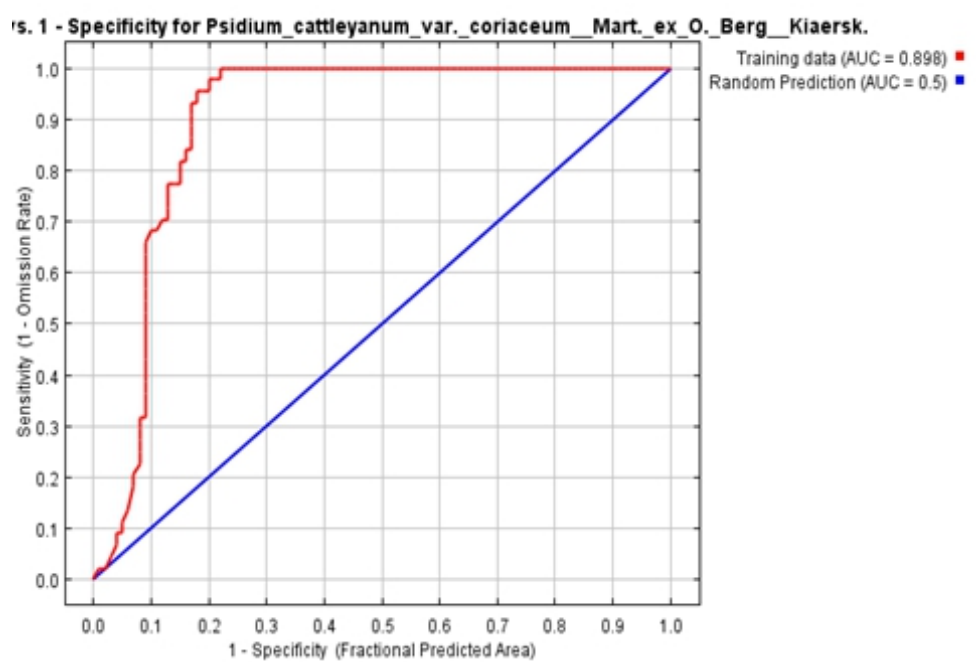

Figure 3. Courbe de ROC « Receiver Operating Characteristic » issu du modèle

\section{Usages de l'espèce.}

$P$. cattleyanum var. coriaceum fait partie des plantes à usages multiples introduites dans la forêt du Karthala et qui sont devenues pratiquement autochtones. Elle figure parmi la liste des 30 espèces les plus préférées par la population locale tous usages et toutes considérations confondus. Elle est utilisée comme tuteur dans la culture de la vanille (Photos 5).

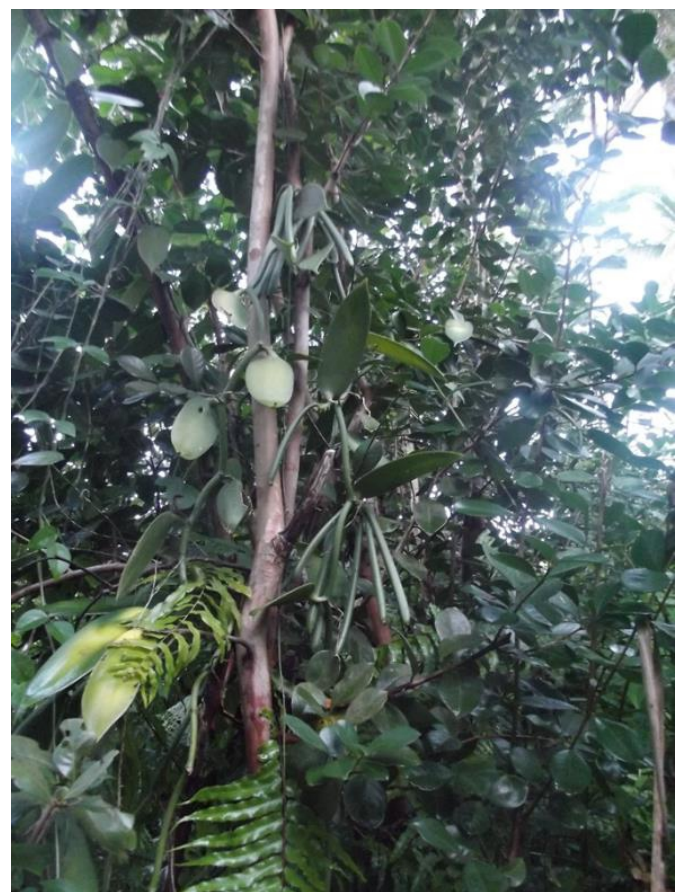

Photo 5. P. cattleyanum Tuteur pour la culture de vanille 
Dans certains usages, elle est la plus préférée des populations. C'est le cas de la production de charbon où elle devance Nuxia pseudodentata et Mangifera indica (Charahabil, 2011). Le Tableau 2 résume l'importance de l'espèce dans les différents usages.

Tableau 1. Fréquence relative du P. cattleyanum dans différents usages.

\begin{tabular}{|c|c|c|c|}
\hline Usages & $\begin{array}{c}\text { Fr de citation } \\
(\%)\end{array}$ & $\begin{array}{c}\text { Parties } \\
\text { utilisées }\end{array}$ & Observation \\
\hline Bois de feu & 33,1 & Tronc & Excellent charbon \\
\hline Bois de service & 3,3 & Tronc & $\begin{array}{c}\text { Construction des cases et dans la } \\
\text { maçonnerie }\end{array}$ \\
\hline Alimentation & 2,8 & Fruits & Consommé cru ou en confiture \\
\hline Fourrage & 0,7 & Feuilles & Consommées par les chèvres \\
\hline Santé animale & 1,6 & Jeunes feuilles & $\begin{array}{c}\text { Traite les maladies diarrhéique } \\
\text { des bovins }\end{array}$ \\
\hline
\end{tabular}

En dehors du bois, $P$. cattleyanum n'est pas très sollicité par les populations locales. En termes de fruits sauvages par exemple, les espèces comme Persea americana, Litchi sinensis et Annona muricata sont les plus préférées des populations. Aussi, avec la rareté du bois de certaines espèces comme Weinmannia comorensis, Nuxia pseudodentata, Kaya madagascariensis Gyrostipula comorensis et Macaranga boutonioides que la population s'est rabattu sur son bois pour la construction des habitations.

\section{Discussion}

\section{Structuration de la forêt du karthala en Grande-comore et bref historique} des invasions biologiques

Avant l'arrivée de l'homme, la forêt occupait l'ensemble de la Grande-Comore. Pour s'installer, l'homme a déboisé les zones côtières et les formations des basses altitudes, facilement accessibles pour construire ses habitations et faire de l'agriculture vivrière. L'anthropisation excessive de la forêt, qui a façonné en partie sa structure actuelle, a commencé avec l'arrivée des planteurs de La Réunion qui sont venus développer la monoculture de la canne à sucre (Saccharum officinarum), et accessoirement celles du café (Coffea spp.) et du cacao (Theobroma cacao). Les villageois se sont vus déplacés vers les pentes avec quelques lopins de terre appelés Djivas en langue locale et qui constituent des formations agroforestières à multiples enjeux (Torquebiau, 2002). On trouve dans ces formations des arbres fruitiers telles que Cocos nucifera (cocotier), Mangifera indica (manguier), Artocarpus communis J.R. Forst. \& G. Forst (arbre à pain) et Artocarpus heterophyllus Lam (Jacquier), des espèces du genre Citrus (agrumes)... Ces arbres sont associés à des cultures vivrières telles que le manioc (Manihot esculenta), la tomate (Licopersicum lycopersicum), le pois d'Angole (Cajanus Cajan) et de rentes notamment la vanille (Vanilla planifolia) et 
l'ylang-ylang (Cananga odorata). On y pratique aussi élevage des petits ruminants comme les chèvres et les moutons (Kader, 1986). Au-dessus des habitations et des Djivas ont retrouvé les Uralés, endroits où l'on pratiquait la culture de la canne à sucre, du giroflier en association avec des cultures vivrière comme les pois d'Angole (Cajanus cajan L) et manioc. Ces Uralés sont situés dans les pentes pierreuses et les reliefs accidentés de faible altitude près de la forêt naturelle. Pour faire fonctionner l'industrie de la canne à sucre, les usines coupaient beaucoup de bois de feu dans les forêts alentour (Pascal, 2002). Ces actions ont entrainé un déséquilibre dans l'organisation et la structuration de la forêt, ce qui a favorisé la prolifération des espèces envahissantes. Vos 2004, avait listé 16 espèces ligneuses envahissantes dans l'ensemble de l'archipel des Comores tel Syzygium aromaticum, Syzygium jambos, P. cattleyanum, Albizzia glabereima ect.. Ces espèces cohabitent avec des herbes exotiques tout aussi envahissantes telles Clidemia hirta, Trisetma virusanum, et Scleria novae-hollandiae. Les résultats présentés dans ce travail contribueraient à mieux comprendre le mécanisme d'invasion du $P$. cattleyanum et la problématique de sa gestion.

\section{- Les Données d'inventaires}

L'étude de l'importance écologique (IVI : valeur d'importance écologique) des espèces dans la zone étudiée qui représente la somme de la fréquence relative, de la densité relative et de la dominance relative (Charahabil et al., 2011) a montré que $P$. Cattleyanum figure dans liste des dix espèces les plus importantes de la forêt. Elle est en quatrième position derrière Weinmannia comorensis, Eugenia aromatica et Nuxia pseudodentata. L'étude de la fréquence spécifique a montré que sur les 78 relevés réalisés plus de la moitié héberge Psidium cattleyanum. Dans les forêts des basses altitudes, cette espèce domine l'essentiel du paysage entre 800 et $1000 \mathrm{~m}$ d'altitude (Charahabil et al., 2013). Elle forme une bande bien délimitée qui parcourt la forêt d'est en ouest pratiquement à égale altitude. Dans les latitudes plus basses correspondantes aux champs et aux parcours des villageois tous les espaces libres sont pratiquement remplacés par cette espèce.

\section{- Le potentiel de régénération du $P$. cattleyanum}

Il n'existe pas encore dans la littérature de données quantitatives sur la production de fruits et de graines par la variété comorienne du $P$. cattleyanum. Comparés aux autres espèces autochtones les mieux représentées dans la forêt, les résultats montrent que $P$. Cattleyanum présente un potentiel de régénération largement plus importante. Les faibles taux de régénération de $O$. comoriensis, $T$. comorensis, $W$ comorensis et $A$. theaeformis indiquent que ces espèces autochtones sont vieillissantes et que leurs niches écologiques tendent à être occupées par $P$. Cattleyanum. Carrière et al. 2008, ont révélé, sur l'espèce malgache, qu'un pied produit en moyenne 
90 fruits par saison $( \pm 37)$ et jusqu'à 162 fruits pour certaines tiges. Un fruit de $P$. cattleianum produit en moyenne 21 graines $( \pm 5)$. Ce travail n'a pas été en mesure de compter ce nombre de fruits par arbre. Cependant, les observations de terrains ont montré que sur cinquante fruits étudiés la moyenne est de 30,31 graines par fruit. Sur 10 pesées effectués, le nombre de fruits par kilogramme est en moyenne de 209,6. Cette grande quantité de fruits et de graines additionnée au nombre important d'oiseaux frugivores (Louette, 1997), de la forêt favorise considérablement l'invasion de cette espèce dont la dissémination ce fait essentiellement par endozoochorie.

\section{- La structure horizontale du $\boldsymbol{P}$. cattleyanum}

La connaissance de la distribution des diamètres d'un peuplement est d'un grand intérêt pour l'aménagement durable des peuplements végétaux (Fonton \& Sagbo, 2004). Elle permet une meilleure évaluation des ressources immédiatement disponibles et la planification future des prélèvements. L'allure en $U$ de la courbe de distribution montre une dominance des individus des deux premières classes $(10-20 \mathrm{~cm}, 20-30 \mathrm{~cm})$ et de la dernière classe $(\geq 100 \mathrm{~cm})$. Ce résultat indique que le diamètre minimum exploitable pour le bois est donc de $30 \mathrm{~cm}$. Les individus au-delà de cette circonférence ne surviennent que très peu. Les individus de gros diamètres sont maintenus pour leur production de fruits, leur ombrage, et pour d'autres usages.

- Usages du P. cattleyanum

Une espèce est dite à usages multiples lorsqu'elle est utilisée dans plusieurs besoins (Mollet et al. 2000). Il peut s'agir soit d'une seule partie de la plante qui est utilisée dans différents besoins, soit de différentes parties de la plante, chaque partie pour un usage bien défini. Les résultats de l'enquête ont montré que $\mathrm{P}$ cattleianum plusieurs catégories d'usages (4 sur les 6 catégories) : Les fruits sont consommés dans l'alimentation humaine et la pharmacopée traditionnelle, les feuilles utilisées dans la pharmacopée et l'alimentation animale et le bois est utilisé dans la production de charbon et dans l'artisanat. L'arbre est aussi utilisé comme tuteur de la culture de vanille pour sa solidité et sa disponibilité.

\section{Stratégie de gestion de l'invasion}

Il est aujourd'hui connu que la solution (et la moins coûteuse) pour éliminer une espèce envahissante est de s'y attaquer dans sa phase d'installation avant le début de son extension (McDonald 1990, Hobbs \& Humphries, 1995). Il existe cependant en Grande-Comore des conflits d'intérêts du fait que cette espèce est largement utilisée par la population. De plus, elle est extrêmement difficile à arracher après le stade jeune. Les jachères de la forêt de la Grille et du Karthala sur lesquelles ces pieds sont originellement gardés pour leurs fruits sont aujourd'hui difficilement remises 
en culture (Vos, 2004). Ils évoluent vers un taillis mono spécifique impénétrable comme à Madagascar (Carrière, 2008).

Pour réduire sa vitesse d'invasion, il faut réduire sa capacité de dissémination. Or, le manque de structure de transformation et de conservation des nombreux fruits produits chaque année, fait que les oiseaux, disséminateurs par excellence, consomment plus de fruits que les hommes qui ne peuvent en cueillir plus qu'est-ce qu'ils peuvent consommer à l'immédiat. Le reste est pourri au sol et les graines germent soit sur place soit transportée dans l'estomac des animaux et des oiseaux vers d'autres sites. La forêt du Karthala étant connue pour sa grande diversité d'oiseaux frugivores et granivores (Louette,1997), la dissémination est très importante. La gestion intégrée, qui est la plus adéquate dans ce type de situation passerait donc par un développement du secteur artisanal ou industriel qui mettrait en place des unités de conservation et de transformations des fruits.

Il faut aussi réduire la dépendance des populations vis-à-vis du bois de l'espèce. Cette dépendance fait que dans certains endroits cette espèce est cultivée. En effet, les ménages comoriens utilisent, pour sa grande majorité, le bois comme source d'énergie. Le manque d'électricité et de gaz en milieu rural et parfois même en milieu urbain, fait du charbon l'unique voie de secours. Or, les enquêtes ont révélé que les trois espèces les plus utilisées sont respectivement $P$. cattleyanum, $M$. indica et $N$. pseudodentata. Le manguier est le plus souvent épargné du faite que ses fruits portent plus d'intérêts pour les populations que le bois. $N$. pseudodentata se présente en population vieillissante et le bois devient de plus en plus rare. En plus son charbon est très peu résistant et se brule trop vite (Charahabil, 2011).

Ainsi, tant qu'il n'y aura pas de politique énergétique fiable aux Comores, les populations auront toujours besoin de charbon et laisseront proliférer $P$. cattleyanum ou même le cultiver comme on le fait à Mohéli (Vos, 2004).

\section{Conclusion}

Une lutte efficace contre l'invasion biologique passe par des connaissances assez solides sur le comportement et le mécanisme d'invasion d'espèces envahissantes. Or, aux Comores, ces données sont presque inexistantes. Ce travail a tenté de mettre en évidence le mécanisme d'invasion du $P$. cattleyanum à travers les données structurales quantitatives de sa population une modélisation de sa niche écologique. Les données structurales quantitatives de sa population ont montré sa forte densité, son fort potentiel de régénération et sa dynamique progressive. Les enquêtes ethnobotaniques ont révélé que cette espèce est fortement sollicitée par la population pour son bois et pour d'autres usages. 
Le cas d'invasion de l'espèce étant régional avec des variétés qui ne se comportent pas de la même façon, il serait intéressant de mener une étude de comparaison entre les différentes îles du sud-ouest de l'océan indien afin de mieux définir des stratégies communes de gestion et de lutte contre l'envahissement de cette espèce.

\section{References:}

1. Baider, C., \& Florens, F.B. (2011). Control of invasive alien weeds averts imminent plant extinction. Biological Invasions, 13, 26412646.

2. Binggeli, P., Hall, J. B., \& Healey, J. R. (1998). An overview of invasive woody plants in the tropics. An overview of invasive woody plants in the tropics, (13).

3. Brown, K.A. et Gurevitch, J., (2004). Long-term impacts of logging on forest diversity in Madagascar, Proceedings of the National Academy of Sciences, 101, 6045-6049. DOI: 101,073/pnas.0401456101

4. Campbell D.G., Hammond H.D. (eds) (1989) Floristic inventory of tropical countries: the status of plant systematics, collections, and vegetation, plus recommendations for the future /. P.imprenta: Bronx, N.Y... New York Botanical Garden,. c1989.. 545 p. : 29 cm.

5. Carrière S.M., Andrianotahiananahary H., Ranaivoarivelo N., Randriamalala J. (2005). Savoirs et usages des recrus post-agricoles du pays betsîleo : valorisation d'une biodiversité oubliée à Madagascar. VertigO, Vol. $6 \mathrm{~N}^{\circ} 11$.

6. Carrière, S. M., Randrianasolo, E., \& Hennenfent, J. (2008). Aires protégées et lutte contre les bioinvasions : des objectifs antagonistes? Le cas de Psidium cattleianum Sabine (Myrtaceae) autour du parc national de Ranomafana à Madagascar. VertigO-la revue électronique en sciences de l'environnement, 8 (1).

7. Fleischmann, K. (1997). Invasion of alien woody plants on the islands of Mahé and Silhouette, Seychelles. Journal of Vegetation Science, 8 (1), 5-12.

8. Fonton, H. N., \& Sagbo, A. A. P. (2004). Modélisation des distributions des paramètres dendrométriques d'une espèce ligneuse dominante en peuplement naturel. Annals of forest science, 61 (6), 545-549.

9. Gounot M., (1969). Méthodes d'étude quantitative de la végétation. Ed Masson et Cie, Paris, 314 p.

10. Hobbs, R.J. \& Humphries, S.E. (1995). —An integrated approach to the ecology and management of plant invasions. Conservation Bio/ogy, 9: 761-770 
11. Huenneke, L. F., \& Vitousek, P. M. (1990). Seedling and clonal recruitment of the invasive tree Psidium cattleianum: implications for management of native Hawaiian forests. Biological Conservation, 53 (3), 199-211.

12. Charahabil M. M. (2011). Caractéristiques de la flore et de la végétation ligneuse du Karthala (Grande-Comore). Thèse de Doctorat, École Doctorale Science de la vie de la Santé et de l'environnement SD-EV. UCAD.

13. Charahabil, M. M., Yahaya, I., Labat, J. N., \& Akpo, L. E. (2013). Typology of the woody synusium of the Karthala forest, Comoro Archipelago. Scripta Botanica Belgica, 50, 100-110

14. Liszkowski, H. D. (2000). Mayotte et les Comores : escales sur la route des Indes aux XVe et XVIIIe siècles. Editions du Baobab.

15. Lorence, D. H., \& Sussman, R. W. (1986). Exotic species invasion into Mauritius wet forest remnants. Journal of Tropical Ecology, 2 (2), 147-162.

16. Louette M. (1996). Biogéographie, origine et évolution des oiseaux aux Comores. In: Lourenço W.R. (ed.) Biogéographie de Madagascar = Biogeography of Madagascar. Paris: ORSTOM, 337-348. (Colloques et Séminaires). Biogéographie de Madagascar : Colloque International, Paris (FRA), 1996/09/26-28. ISBN 2-7099-1324-0

17. Mcdonald, M. (1989). The Forest in the Trees: Collective Rights as Basic Rights. Lafrance, G. (éd.). Ethique et droits fondamentaux/Ethics and Basic Rights. Ottawa: Les Presses de l'Université d'Ottawa, 1989, p. 230-241.

18. Mollet M. Téré H., Herzog F. (2000). Ligneux à usages multiples dans les systèmes agraires tropicaux : une étude de cas de Côte d'Ivoire. Schweiz. Z. Forstwes. 151 (2000) $10: 355-364$.

19. Normand, F. (2002). De la fleur au fruit : étude et modélisation de la floraison, de la fécondation-fructification et de la croissance du fruit chez le goyavier-fraise (\# Psidium cattleianum\#).

20. Perneger, T. Perrier, A. (2004). Courbe ROC, ou « receiver operating characteristic ». $\quad$ http://www.em-consulte.com/article/144229 [consulté le 08/12/2010].

21. Philips O., Gentry A. H. (1993). The useful plants of Tambopata, Peru. II. Statistical hypothesis tests with a new quantitative technique. Economic Botany, 47 (1) : 33-43

22. Rioult F. (2011). Interprétation graphique de la courbe ROC. eric.univlyon2.fr/ ricco/cours/slides/roc_curve.pd. Consulted le 28/10/2016.

23. ROMBI, M.-F. (2018.). Les Comoriens: Africains et Orientaux. Consulté le 02 28, 2018, sur Encyclopedia Universalys : https://www.universalis.fr/encyclopedie/comores/2-histoire/ 
24. Sghaier, T., \& Palm, R. (2002). Répartition des arbres et des volumes par classes de grosseur dans les peuplements de pin d'Alep (Pinus halepensis Mill.) en Tunisie. Annals of forest science, 59 (3), 293-300.

25. Stéphanie M. C, Eric Randrianasolo et Julie H., (2018). "Aires protégées et lutte contre les bioinvasions : des objectifs antagonistes? Le cas de Psidium cattleianum Sabine (Myrtaceae) autour du parc national de Ranomafana à Madagascar.", VertigO - la revue électronique en sciences de l'environnement [En ligne], Volume 8 Numéro 1 | Avril 2008 mis en ligne le 12 avril 2008, consulté le 25 février 2018. URL : http://journals.openedition.org/vertigo/1918; DOI : 10.4000/vertigo.1918

26. Tassin J, Triolo J, Blanfort V et Lavergne C. (2009 b). L'évolution récente des stratégies des invasions végétales de l'île de la Réunion. Rev. Ecol. (Terre vie). Vol. 64. http://hdl.handle.net/2042/55779

27. Tassin, J., Bellefontaine, R., Roger, E., \& Kull, C. A. (2009 a). Évaluation préliminaire des risques d'invasion par les essences forestières introduites à Madagascar. BOIS \& FORETS DES TROPIQUES, 299 (299), 27-36.

28. Tassin, J., Lavergne, C., Muller, S., Blanfort, V., Baret, S., Le Bourgeois, T.,... \& Rivière, J. N. (2006). Bilan des connaissances sur les conséquences écologiques des invasions de plantes à l'île de la Réunion (Archipel des Mascareignes, Océan Indien).

29. Torquebiau, E., Mary, F., \& Sibelet, N. (2002). Les associations agroforestières et leurs multiples enjeux. BOIS \& FORETS DES TROPIQUES, 271 (271), 23-35.

30. Vitousek, P. M. et Walker, L. R., (1989). Biological invasion by Myrica faya in hawaii: plant demography, nitrogen fixation, ecosystem effects, Ecological Monographs, 59, 247-265.

31. Vos, P. (2004). Etudes de plantes ligneuses envahissantes de l'archipel des Comores (Union des Comores et Mayotte).

32. Zainaba A. (2009). Contribution à l'étude de la pharmacopée traditionnelle des Comores : Enquête ethnobotanique dans la région de Hamahame. Thèse de Doctorat en Pharmacie Université Cheikh Anta Diop de Dakar/Sénégal. 128 p. 